\title{
Filigrane
}

Écoutes psychothérapiques

\section{Comment retrouver la dignité de penser dans une société d'automatismes?}

\section{Roland Gori}

Volume 21, numéro 2, automne 2012

Psychanalyse et engagement II

URI : https://id.erudit.org/iderudit/1015196ar

DOI : https://doi.org/10.7202/1015196ar

Aller au sommaire du numéro

Éditeur(s)

Revue Santé mentale au Québec

ISSN

1192-1412 (imprimé)

1911-4656 (numérique)

Découvrir la revue

Citer cet article

Gori, R. (2012). Comment retrouver la dignité de penser dans une société d'automatismes ? Filigrane, 21(2), 27-40. https://doi.org/10.7202/1015196ar
Résumé de l'article

À partir des deux principales significations du mot « engagement ", celle qui fait du sujet l'acteur d'une promesse et celle qui le voue à la contrainte des automatismes, l'auteur évoque les logiques de domination et d'émancipation sociale de la civilisation actuelle. Il montre comment les formes de savoir et les formes de pouvoir sont inséparables et propose la thèse selon laquelle la récusation de la psychanalyse aujourd'hui dépend étroitement de son appartenance au savoir narratif. 


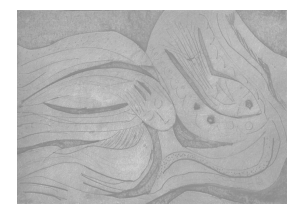

\title{
Comment retrouver la dignité de penser dans une société d'automatismes?
}

\author{
Roland Gori
}

\begin{abstract}
À partir des deux principales significations du mot "engagement", celle qui fait du sujet l'acteur d'une promesse et celle qui le voue à la contrainte des automatismes, l'auteur évoque les logiques de domination et d'émancipation sociale de la civilisation actuelle. II montre comment les formes de savoir et les formes de pouvoir sont inséparables et propose la thèse selon laquelle la récusation de la psychanalyse aujourd'hui dépend étroitement de son appartenance au savoir narratif.
\end{abstract}

En résumé, l'arithmétique des plaisirs enlève à la vie le ressort même du plaisir, c'est-à-dire la capacité subjective d'invention, de création, d'imprévu. Avec le risque disparaît aussi le sel de la vie. C'est une morale de commerçant prudent. (Canguilhem, 2011, 117)

\section{Le sentiment linguistique}

e «sentiment linguistique», comme le nomme Freud (1939/1986) dans L'homme Moïse et la religion monothéiste, constitue pour les praticiens que nous sommes un guide précieux dans l'acte clinique comme dans la recherche théorique. Le mot «engager» est «formé de «gage» avec le préfixe en-; on relève en latin médiéval les formations analogues se ingnadiare «s'engager (à fournir des preuves)» et invadiare "mettre en gage» (Rey, 1992). Le verbe en français reprend cette idée de contrainte, d'obligation et de dette pour désigner l'action de "mettre en gage », c'est-à-dire de donner «en garantie» ou en caution. Recevoir un engagement, c'est accepter la reconnaissance d'une dette, se lier par une promesse ou un serment. C'est un lien fort, plus ou moins librement consenti, qui place celui qui s'y engage dans un état d'obligation, de promesse. L'engagement de la parole dans la promesse révèle la dette qu'elle contient, dévoile la responsabilité sociale autant 
que subjective de celui qui la prononce. C'est une parole performative, au sens d'Austin (1970), qui réalise ce qu'elle dit, qui montre ce qu'elle énonce, action autant que discours, elle contraint celui qui la tient autant qu'elle le tient. À moins que le sujet ne se dérobe aux obligations qu'implique sa promesse, quitte à déchoir de la valeur que l'on accorde aux personnes « dignes de parole», aux gens que l'on «croit sur parole».

À partir du XVI e siècle, le terme « engager » prend un sens figuré de «faire pénétrer dans (quelque chose qui ne laisse pas libre)»(Rey, 2005). L'engagement prend alors valeur de privation de liberté, d'entrainement dans un processus ou un chemin qui contraint à en suivre le cours. Le sujet se trouve engagé dans une situation qui le fait entrer dans une action, un lieu ou un objet qui le contraignent dans son entreprise. L' « engagement» se rapproche alors de l'automatisme du destin, de la fatalité ou du choix décisif. Il est parfois possible de «rebrousser» chemin d'un lieu dans lequel on s'est engagé, mais c'est bien en tant qu'à le poursuivre nous serions conduits dans l'erreur ou la méprise. À partir de l'époque classique, ce terme «engagement» désigne ce qui pousse à agir d'une certaine façon, tout autant que l'état de la personne liée à une autre dans une situation sentimentale ou sociale: du contrat de travail à la promesse de mariage en passant par l'entrée dans une administration militaire (Rey, 2005).

Tous les autres emplois du terme «engager» se déduisent de ces deux valeurs. D’une part le sens du mot est celui de «s'obliger soi-même» par une parole, un contrat, bref une promesse, et d'autre part le deuxième sens est davantage de "se trouver obligé » par un automatisme qui encastre, dans un état qui « engage une chose dans une autre» (Ray, 2005). Le terme s'emploie aussi bien pour désigner l'encastrement d'une porte dans un lieu que celui de la position d'une roue dans une crémaillère ou encore l'émergence de «la tête du fotus dans l'excavation pelvienne»(Rey, 2005). De cette signification dérive au sens figuré l'idée d'une prise de position, conduisant à parler d'artistes ou d'intellectuels «engagés».

Il m'a semblé intéressant de rappeler ces deux valeurs fondamentales du terme « engagement» - dont découlent toutes les autres - celle d'un sujet actif de la dette et de la promesse, celle d'un sujet passif, contraint par autre chose que lui-même, engagé dans un «défilé » qui le dépossède de sa liberté, pour évoquer aujourd'hui les logiques de domination et d'émancipation sociales autant que subjectives. Le sujet engagé par sa parole dans le débat politique est un citoyen subjectivement responsable. Le sujet engagé dans les dispositifs de normalisation sociale est un sujet conformiste, consommateur et 
spectateur de ses jouissances existentielles. Cet article rappelle brièvement les thèses exposées dans l'ouvrage La dignité de penser. En particulier je voudrais rappeler que les logiques de soumission et d'émancipation sociales s'avèrent tributaires des formes $d u$ savoir à un moment donné et dans une civilisation donnée. Et réciproquement, que les formes du savoir sont les formes du pouvoir.

\section{Les formes du savoir sont les formes du pouvoir}

Quand je dis que les formes du savoir à une époque donnée et dans une société donnée sont inséparables des formes de pouvoir, des pratiques sociales en œuvre à ce moment-là, cela ne veut pas dire que les découvertes scientifiques soient de pures constructions sociales - conception aussi absurde que dangereuse -, mais que la culture, dont elles émergent, favorise ou inhibe leur émergence et leur développement.

C'est en ce sens qu'avec Michel Foucault j'avance la thèse selon laquelle la psychopathologie est aussi un «fait de civilisation» (Foucault 1954/2005; Gori et Del Volgo, 2005/2009). Autrement dit, les objets, les concepts et les pratiques des psychiatres et des psychologues ont une histoire, histoire qui révèle des styles de société (Gori et Del Volgo, 2008). Dans la manière de s’y prendre avec la souffrance psychique des êtres humains qui la composent, une société révèle ses valeurs, sa substance éthique. Mais ce serait une grossière erreur de penser que la situation qui est celle de la psychanalyse ou de la psychopathologie aujourd'hui, dans son embarras avec le pouvoir, est une exception. Ce n'est qu'un cas particulier du principe selon lequel les formes du savoir et les formes du pouvoir communiquent entre elles, sont consubstantielles aux valeurs éthiques d'une société qui les évalue davantage pour leur «utilité sociale» que pour leurs progrès scientifiques ou thérapeutiques réels.

Pour exemple, l'historien de la médecine Henry Sigerist (1932) montre que la découverte de la physiologie de la circulation par Harvey est inséparable de l'histoire intellectuelle de l'Europe au début du XVII ${ }^{e}$ siècle. Sigerist montre que c'est l'épanouissement du baroque qui donne à la science médicale ce point de vue perspectiviste ouvert à l'illimité et l'infini, lui permettant de passer du modèle anatomique à l'idéal physiologique. C'est la profonde transformation dans la façon de considérer le monde qui trouve son premier écho dans les arts plastiques avec l'épanouissement du baroque, qui recompose le savoir médical au profit de ce point de vue perspectiviste. Sigerist écrit : «L'homme du baroque ne s'intéresse pas à ce qui est, mais à ce qui va être. 
Le baroque est infiniment plus qu'un style dans l'art; il est l'expression d'une forme de pensée qui règne à cette époque dans tous les domaines de l'esprit : la littérature, la musique, la mode, l'État, la façon de vivre, les sciences. [...] On peut exactement porter à son compte [le médecin] ce que Wölfflin a dit de l'artiste: il ne voit pas l'œil humain, mais seulement son regard. Lui aussi ne s'attachera plus au corps dans son harmonie figée, mais au mouvement sans entraves de l'organisme dans son ensemble et de ses diverses parties. » (Sigerist, 1932, 41) Il précise : «la physiologie, l’idée fonctionnelle en médecine, est née de l'esprit baroque [...]. Nous voyons que la médecine est des plus étroitement liée à l'ensemble de la culture, toute transformation dans les conceptions médicales étant conditionnée par des transformations dans les idées de l'époque ${ }^{1}$.» (Sigerist, 1932, 42) Ainsi la découverte de la physiologie de la circulation sanguine, loin d'être une construction sociale et culturelle, n'a émergé que parce que les conditions sociales et culturelles de l'époque le permettaient.

J’ai également souligné (Gori 2010, 2011) que la naissance de la démocratie en Grèce au V viècle avant J.-C., se révèle inséparable du développement de la pensée rationnelle, abstraite, mathématique. Mais cette pensée rationnelle des Grecs s'est trouvée elle-même conditionnée par des pratiques sociales. La transformation des pratiques sociales des Grecs qui s'étend du $\mathrm{VI}^{\mathrm{e}}$ au $\mathrm{IV}^{\mathrm{e}}$ siècle avant J.-C., ne concerne pas seulement la vie politique. Cette rationalité qui émerge entre le $\mathrm{VI}^{\mathrm{e}}$ et le $\mathrm{V}^{\mathrm{e}}$ siècle en Grèce se trouve mise en acte dans des pratiques sociales de la Cité, pratiques fondatrices de la démocratie. Cette isonomie ${ }^{2}$ fait de chaque citoyen un "semblable», appelé à participer librement à la gestion des affaires politiques, à égale distance du centre de ce cercle nommé Agora. Mais cette isonomie est aussi un paradigme pour penser le monde, le rapport à soi-même et aux autres. C'est une "catégorie» au sens fort du terme, donnant littéralement un style de présence au monde, à soi-même et aux autres dans tous les secteurs de la pensée et de la vie humaine. Cette forme de rationalité permet de penser l'ordre du monde physique, social et humain selon le même modèle: rapport de symétrie, d'équilibre et d'égalité entre les différents éléments qui les composent. Jean-Pierre Vernant écrit que «de fait, c'est sur le plan politique que la Raison, en Grèce, s'est tout d'abord exprimée, constituée, formée [...] en fournissant aux citoyens le cadre dans lequel ils concevaient leurs rapports réciproques, la pensée politique a[-t-elle] du même coup orienté et façonné les démarches de leur esprit dans d'autres domaines» (Vernant, 1962/2007, 131-132). La Raison fut d'abord politique. La philosophie, la logique, les mathématiques, la 
physique, la justice, l'art, la médecine, l'architecture, se sont transformés en miroir des pratiques sociales qui tendaient au quotidien à s'imposer dans la Cité.

Mais qu'en est-il aujourd'hui des formes du savoir dans notre civilisation?

Aujourd'hui la nouvelle colonisation des esprits passe par l'extension du langage de l'économie, de ses valeurs, de sa fonctionnalité, de ses caractères quasi anonymes, abstraits et sans expressivité, pour abolir les particularismes culturels des classes sociales et nier chaque subjectivité. Dans le champ qui est le nôtre, la psychopathologie, les savoirs et les pratiques s'alignent sur les exigences de ce paradigme culturel, des modes de production du sujet éthique qu'il engage. Quel est-il?

L'humain se transforme en «capital» (Becker, 1964/1994) que l'on doit exploiter comme "ressources », et auquel on apprend à "gérer » ses émotions, son deuil, ses «habiletés sociales», ses «compétences cognitives», sous prétexte d'accroitre ses «performances» et sa «compétitivité». La vie devient un champ de courses avec ses "handicaps", ses départs, ses "deuxièmes chances» et son arrivée. À un tel point que la notion de «handicap» tend à envahir tous les champs: celui de l'école, de la psychiatrie, de la psychologie, de la médecine, du travail social, de l'économie, de la sociologie... Mais d'où vient ce mot? Le terme provient de l'anglais " hand in cap», «la main dans le chapeau », primitivement jeu de hasard appliqué ensuite aux courses de chevaux au XVIII ${ }^{e}$ siècle. Le terme "handicap " a été introduit en français «avec l'idée d'égaliser les chances des concurrents en imposant aux meilleurs de porter un poids plus grand ou de parcourir une distance plus longue. Par extension, le terme [...] se dit de tout désavantage imposé dans une épreuve à un concurrent de qualité supérieure. De là vient [...] le sens figuré d'« entrave, gêne», «infériorité»». Le participe passé du verbe "handicaper», d'abord dans le domaine hippique et ensuite dans le champ social désigne une personne désavantagée, et notamment une personne désavantagée par une déficience physique ou mentale. C'est un concept très intimement lié à l'esprit de compétition établissant l'idée de jugement comparatif de la valeur des objets, des chevaux puis des personnes.

Définir la souffrance d'un individu à la lumière de ses chances à concourir dans le champ social participe d'une civilisation sportivo-managériale des mœurs (Gori et Del Volgo, 2008) qui fait de la vie un champ de course. La reconnaissance sociale du handicap, notamment "psychique», pour essentielle qu'elle soit du point de vue de la protection sociale des individus vulnérables et de leurs droits à la dignité et à l'accompagnement, n'est pas sans 
conséquence sur la recomposition des savoirs et des pratiques de la psychopathologie. Cette conception de l'humain engage.

C'est du point de vue de la psychopathologie comme «fait de civilisation» (Foucault, 1954/2005) que j'interroge la notion de «handicap», afin de rendre compte du dispositif dans lequel aujourd'hui sont traitées les souffrances psychiques et sociales. J'entends «dispositif» au sens fort du terme, tel que Giorgio Agamben le définit après Foucault: "tout ce qui a d'une manière ou d'une autre, la capacité de capturer, d'orienter, de déterminer, d'intercepter, de modeler, de contrôler et d'assurer les gestes, les conduites, les opinions et les discours des êtres vivants.» $(2006 / 2007,31)$ Le dispositif présente pour Foucault une nature et une fonction essentiellement stratégiques qui supposent des interventions dans les jeux de pouvoir par des types de savoir dont ils sont à la fois l'occasion, la conséquence et l'origine. Ce sont les modes concrets par lesquels un savoir et un pouvoir communiquent pour fabriquer un sujet éthique. Ce sont les appareils hétérogènes d'un pouvoir qui disposent les individus à subjectiver leurs comportements, leurs gestes et leurs pensées selon le modèle de civilisation d'une société et d'une époque. Comme l'écrit encore Giorgio Agamben, «le dispositif est donc, avant tout, une machine qui produit des subjectivations et c'est par quoi il est aussi une machine de gouvernement» $(2006 / 2007,42)$.

Nos diagnostics en psychopathologie en disent au moins autant sur la «substance éthique» d'une culture que sur la souffrance des patients, et davantage encore sur le mode de formation des praticiens qui les prennent en charge. Comment ne pas reconnaître dans le miroir des diagnostics psychiatriques les formes mêmes de la culture dont ils émergent, de ses savoirs et de son éthique? Ces diagnostics sont les « défilés» dans le dispositif desquels le social et le politique engagent les souffrances psychiques des patients pour leur prise en charge. Avec l'expansion des paradigmes actuels de la santé mentale, l'épidémie des troubles du comportement progresse toujours davantage, en particulier au rythme des «nouveaux» médicaments mis sur le marché. Ces médicaments, paradoxalement, fabriqueraient les diagnostics davantage qu'ils ne traiteraient les maladies. Au point que certains auteurs se sont émus de ces manœuvres de manipulation de l'industrie pharmaceutique responsables de l'invention de maladies (Blech, 2003; Hugnet, 2004; Pignarre, 2001, 2003). Qu'il s'agisse des troubles de l'anxiété sociale, des troubles déficitaires de l'attention et de l'hyperactivité, de la dysphorie prémenstruelle, de la dysfonction érectile, des troubles oppositionnels de provocation ou de toutes les nouvelles maladies de l'âme, il s'avère de plus en plus difficile de savoir ce qui 
du diagnostic ou du traitement est premier. Davantage la pathologie se donne dans la flexibilité et la liquidité de ses frontières, davantage la ligne de partage entre le normal et le pathologique se trouve brouillée, davantage encore il est difficile de faire la différence entre la prescription « thérapeutique » et la prescription «cosmétique » ou sociale - le «deuil » est-il un épisode existentiel à traiter comme un état dépressif? Dès lors que chaque culture dessine un profil de la maladie mentale, celui-ci se révèle comme le reflet inversé de la conception, non seulement de la santé, mais aussi du style anthropologique de la société dont il émerge. À ce titre, la notion de handicap "psychique» participe d'une civilisation des mœurs qui tend à brouiller les frontières entre l'«anomalie» et le «pathologique», non sans offrir, il est vrai, une consolation, une compassion sociale aux patients qu'elle prend dans son dispositif. Il ne s'agit pas de contester les conséquences heureuses de ce dispositif, il s'agit plutôt de se demander quelles peuvent être les conséquences épistémologiques et de quelle culture politique ce concept est le symptôme.

Le «flou » conceptuel de la notion de « handicap ${ }^{3}$ » participe aujourd'hui aussi à cet éclatement des grandes structures psychopathologiques au profit des «troubles du comportement» diagnostiqués notamment par les différentes versions du DSM, passant d'une centaine d'entités psychopathologiques en 1952 (DSM I) à plus de 400 en 1994 (DSM IV) ${ }^{4}$. Cette fluidité et cette flexibilité des diagnostics que permettent des notions comme celles de troubles du comportement ou de handicap psychique, participent au démembrement des grandes structures psychopathologiques et interrogent les concepts de norme, d'anomalie et de pathologie. Et ce d'autant plus que la rationalisation des conduites dans notre civilisation prend toujours davantage la forme d'une médicalisation de l'existence (Gori et Del Volgo, 2005, 2008).

Dans la sourde et discrète matérialité des dispositifs de sécurité, de surveillance et de contrôle social, se noue aujourd'hui un lien toujours plus étroit entre l'individu et le pouvoir. Dans la famille, à l'école, au travail, dans le soin, dans le champ des loisirs, dans celui de la sexualité comme du crime, s'inscrivent insidieusement des nouvelles normes sociales comme autant de produits et d'opérateurs des dispositifs de normalisation (Gori, Cassin et Laval, 2009). Cette société de normalisation se préoccupe moins de sanctionner le crime que de prévenir les risques de délits et de récidives des individus et des populations soupçonnés par la science de devenir dangereux ou déviants. Avec ce risque de pénaliser les problèmes sociaux en les médicalisant et de légitimer les inégalités sociales, la société de normalisation demande toujours plus à la psychiatrie de dire « comment le criminel ressemblait à son crime 
avant même de l'avoir commis" (Foucault, 1999). Aujourd'hui, comme au $\mathrm{XIX}^{\mathrm{e}}$ siècle, le Pouvoir et la psychiatrie ciblent l'enfance, devenue piècecharnière d'un dispositif de gestion des «risques", par le repérage précoce et féroce des conduites et des troubles déviants. Ce n'est plus la maladie mentale qui intéresse cette «nouvelle» psychiatrie, mais tout «ce petit peuple des anormaux » qu'il s'agit de dépister le plus férocement et le plus précocement possible pour rendre compte de leur "trajectoire d'agression physique», quitte à leur ouvrir la «carrière » d'exclus sociaux rendue possible par la prophétie autoréalisatrice des diagnostics « médicaux» (Gori et Del Volgo, 2008).

Cette «nouvelle psychiatrie» se donne pour mission de dépister, d'archiver et de redresser, à l'aide de la chimie et des techniques psychorééducatives, les «troubles du comportement», les individus appartenant à des "populations à risque», dont il faut évaluer le risque de "récidive». Dans une telle civilisation, les sentiments, les relations sociales, leur psychologie et leur psychopathologie tendent toujours davantage à se forger, à s'éduquer, à se corriger, à se juger et se soigner par le langage du commerce et de l'économie (Gori et Le Coz, 2006). D'où dans notre civilisation aujourd'hui ces idéaux d'objectivité, de rationalité, d'efficacité et de probabilité, au sens de preuve que "ça marche», qui sont portés au pinacle des valeurs et qui prévalent sur l'amour de la vérité, le sens de l'honneur, la dignité humaine, le goût de l'autre et l'hospitalité pour le rêve ou la folie. Ce monde des valeurs dominantes dans notre civilisation s'impose non seulement dans la recomposition des champs du savoir ou du soin, mais encore dans celui de la justice, de l'éducation, de la culture et des relations sociales ${ }^{5}$.

L'engagement du sujet dans le social peut ici s'entendre comme un système de contraintes qui le dépouille toujours davantage de sa liberté, de sa responsabilité subjective et citoyenne. Le sujet des «démocraties de la norme» est un sujet toujours plus engagé dans des automatismes qui n'exigent d'autre promesse que celle de sa servitude volontaire (Abelhauser, Gori et Sauret, 2011).

\section{La crise du récit}

Mais comment en sommes-nous venus à transformer le savoir et sa valeur émancipatrice en instrument de soumission sociale? Comment l'engagement du sujet singulier et collectif a-t-il pu passer de la promesse démocratique à la soumission sociale «librement consentie»?

Le pilotage par les chiffres, dans tous les domaines de la vie sociale, marque un passage des discours narratifs de légitimation sociale aux discours 
non-narratifs. Cette transformation de la nature du savoir qui privilégie la part technique, instrumentale du langage - l'information - aux dépens de sa part fabulatrice, de ses fictions et de sa mise en récit, est un fait de civilisation, une machine de gouvernement autant qu'une fabrique des subjectivités. Le sens se perd au profit de la forme, le savoir est traduit et toléré uniquement dans le langage de machine. L'ordinateur qui calcule de manière prodigieuse toutes les données à sa portée, qui réalise merveilleusement toutes sortes d'opérations, ne connaît pas le sens de ce qu'il fait. La connaissance devient une information-marchandise, et la hiérarchie des savoirs qui la composent repose sur la capacité de leurs résultats à être traduits dans ce langage de machine (Lyotard, 1979).

Le déplacement du centre de décision de la pensée du professionnel au programme numérisé des évaluations tend à prolonger l'aliénation de l'ouvrier à la technique de la machine. La conséquence anthropologique des méthodes actuelles de l'exécution du travail est de toujours plus écraser la pensée et la subjectivité des travailleurs (Gori, 2011). Simone Weil dès 1934 en décrit le processus:

Dès lors on se trouve en présence d'une situation paradoxale; à savoir qu'il y a de la méthode dans les mouvements du travail, mais non pas dans la pensée du travailleur. On dirait que la méthode a transféré son siège de l'esprit dans la matière. C'est ce dont les machines automatiques offrent la plus frappante image. Du moment que la pensée qui a élaboré une méthode d'action n'a pas besoin d'intervenir dans l'exécution, on peut confier cette exécution à des morceaux de métal aussi bien et mieux qu'à des membres vivants; et on se trouve ainsi devant le spectacle étrange de machines où la méthode s'est si parfaitement cristallisée en métal qu'il semble que ce soit elles qui pensent et les hommes attachés à leur service qui soient réduits à l'état d'automates (Weil, 1955, 99).

Les machines aujourd'hui sont virtuelles, dématérialisées, façonnant, modelant, capturant les moindres actes symboliques et matériels dans des protocoles et des standards normés, constituant la véritable organisation rationnelle du travail à partir de bases de données et de leurs traitements statistiques. Aujourd'hui c'est l'organisation bureaucratique avec son traitement statistique des données et sa rhétorique d'expertise (Abelhauser, Gori et Sauret, 2011) qui lui donne une légitimité qui permet le développement infini de cette oppression sociale. Cette prolifération de machines numériques comme 
l'hégémonie progressive de l'informatique, promue médiateur quasi exclusif des échanges symboliques entre les hommes et les objets, entre les hommes et leurs systèmes de production et entre les hommes eux-mêmes, a considérablement accru le pouvoir du savoir technicien, et s'est généralisé à l'ensemble des professionnels.

Il ne s'agit plus de savoir si un énoncé est vrai ou juste, mais seulement si son énonciation permet que «ça marche» ou non, plus ou moins. Cet instrumentalisme qui écrase la pensée tend aujourd'hui à occuper le vide tant éthique, politique qu'épistémologique, laissé par l'accélération du désenchantement du monde. Adorno (1951/2003) avait remarqué que cet instrumentalisme n'était pas une forme de pensée, mais tendait à devenir la seule forme dans laquelle une pensée pouvait se former. Les transformations de la parole en "marchandises informationnelles », solubles dans le système numérique, déterminent de nouvelles règles pragmatiques qui constituent les nouvelles formes du lien social et participent à la construction d'un nouvel espace politique remodelant en profondeur le concept de démocratie. C'est donc une nouvelle pragmatique des discours, au sens fort du terme, à la fois épistémologique, éthique et politique qui se met en place dans cette perspective postmoderne, minorant toujours plus le savoir narratif au profit des savoirs techniques.

À suivre cette hypothèse, il apparaît que les savoirs narratifs, en particulier dans le champ des sciences sociales et humaines, mais aussi dans celui des sciences du vivant, se trouvent actuellement dévalorisés ou acculés à devoir se transformer selon les normes des savoirs non-narratifs. À prendre pour seul exemple le champ qui est le nôtre, la psychanalyse serait moins en crise eu égard aux échecs et aux limites qui seraient imputables à sa méthode, qu'en tant que pratique et théorie qui plongent profondément leurs racines dans le genre du savoir narratif. C'est cette familiarité de la théorie et de la pratique psychanalytiques avec le récit qui tendrait à la rendre illégitime aujourd'hui. Rappelons avec Lacan que « [... l'essentiel de la méthode freudienne pour aborder ce qu'il en est des formations de l'inconscient, c'est de se fier au récit. L'accent est mis sur ce fait de langage, d'où tout, à vrai dire, eût pu partir» (Lacan 1969-1970/1991, 72). À partir de cette remarque de Lacan il nous faut bien reconnaitre cette profonde intimité entre la méthode psychanalytique et le savoir narratif, entre la psychanalyse et ce genre du savoir. Intimité que Freud le premier évoque non sans ambivalence lorsqu'il écrit dans les Études sur l'hystérie: «Je m'étonne moi-même de constater que mes observations de malades se lisent comme des romans et qu'elles ne 
portent pour ainsi dire pas ce cachet sérieux, propre aux écrits des savants. » (Freud et Breuer, 1895/1956, 127)

La dette de la psychanalyse à l'endroit du récit n'invalide sa «scientificité » qu'au regard d'une culture qui fait du non-narratif son seul critère de scientificité. À devoir admettre que c'est le récit qui est en crise aujourd'hui dans notre civilisation, c'est autant à une crise de la culture que nous avons affaire qu'à une crise de la psychanalyse en tant qu'elle appartient de pied en cap au savoir narratif. Dans cette nouvelle forme de censure sociale des savoirs, l'art et les «humanités » sont les grands perdants. À nous d'engager les débats tout autant politiques qu'épistémologiques pour qu'ils deviennent les moyens d'une émancipation authentique. Afin que les chiffres nous servent pour parler, non pour nous faire taire, car il convient de rappeler avec Canguilhem que « la raison est régulière comme un comptable; la vie, anarchique comme un artiste» (Canguilhem, 1947, 326).

Aussi importe-t-il, a contrario de l'époque actuelle, de retrouver l'art de raconter et de partager nos expériences. Seuls le récit, l'art, le débat scientifique, le débat politique, avec ce qu'ils permettent du partage de l'expérience et ce qu'ils postulent du principe d'une égalité, peuvent rétablir l'humain dans ses droits ${ }^{6}$. Pour les cliniciens que nous sommes, ce «souci» du récit est vital: «Si l'expression "être de bon conseil" commence aujourd'hui à paraître désuète, c'est parce que l'expérience devient de moins en moins communicable. C'est pourquoi nous ne sommes plus de bon conseil, ni pour nous ni pour autrui. Porter conseil, en effet, c'est moins répondre à une question que proposer une manière de poursuivre une histoire (en train de se dérouler). Pour pouvoir demander conseil, il faudrait d'abord être capable de raconter cette histoire.» (Benjamin, 1972/2000, 119-120) Car le récit est comme ces «graines enfermées hermétiquement pendant des millénaires dans les chambres des pyramides et qui ont conservé jusqu'à aujourd'hui leur pouvoir germinatif » (Benjamin, 1972/2000, 125). Par où il détient cette capacité de nous faire rêver, d'inscrire les événements de vie comme des expériences, expériences de plaisir, de douleur et de joie, mais expériences vivantes d'un sujet qui a pu échapper au trauma de la réification auquel incite notre civilisation et ses contraintes normatives du « rationalisme morbide» (Gori, 2011; Gori et Del Volgo, 2008).

Nous ne reviendrons pas en arrière du système technicien et rien ne nous dit d'ailleurs que ce soit souhaitable. Il convient donc pratiquement et concrètement d'incorporer du savoir narratif, du récit, du mythe, et de l'épopée, au sein de ces dispositifs de normalisation, de contrôle et de traitement de 
l'information. Toute la pratique qui est la mienne montre que cette incorporation du narratif dans le savoir non-narratif est possible à condition et à condition seulement qu'on lutte politiquement pour refuser le désaveu de sa légitimation sociale. Rien n'empêche d'adjoindre à la médecine la plus techniquement sophistiquée une part clinique indispensable qui permette aux patients comme aux soignants de parler, de dire, d'écouter les significations que prennent les situations de souffrance (Gori et Del Volgo, 2005). Rien n'empêche qu'à la construction médico-biologique de la maladie ou des symptômes s'ajoute l'écoute des récits et des «romans» que le patient se trouve contraint d'élaborer pour donner un sens à sa souffrance (Del Volgo, 1997). Rien n'empêche une commission de recrutement, de promotion, d'évaluation des recherches et des formations, de ne pas se limiter aux critères bibliométriques et de débattre de l'ensemble du dossier examiné. Cela ne veut pas dire que nous n'avons plus à traduire en «informations», en «langage de machine» certains évènements lorsque c'est nécessaire, mais que nous posions comme exigence politique minimale d'avoir préalablement à discuter de la nécessité de cette traduction, de dire ses effets et ses limites. Aujourd'hui où les «machines automatiques semblent présenter le modèle du travailleur intelligent, fidèle, docile et consciencieux » (Weil, 1955, 129) et où « dans tous les domaines la pensée, apanage de l'individu, est subordonnée à de vastes mécanismes qui cristallisent la vie collective, et cela au point qu'on a presque perdu le sens de ce qu'est la véritable pensée» (Weil, 1955, 129), il nous faut savoir trouver dans la technique elle-même les germes de l'émancipation sociale à condition et à condition seulement de pouvoir s'opposer à la servitude volontaire qu'elle exige. C'est-à-dire aussi à l'horreur d'une jouissance que le sujet humain éprouve à sa propre chosification, à sa transformation en matière inerte. C'est l'horreur de cette jouissance qui naguère conduisait l'humain aux tâtonnements aveugles d'une servitude volontaire aux princes et aux prêtres et qui aujourd'hui les conduit à accepter l'assujettissement aux machines intelligentes et à ceux qui en garantissent la prétendue objectivité, complices les uns comme les autres de s'abandonner aux logiques de domination. Le courage de penser est ce qui vient faire objection à ce vertige collectif de l'abolition subjective. Ce courage de penser est ce que l'on peut nommer "engagement» ou "dignité», une parole, une promesse qui reconnaît la dette que chacun d'entre nous contracte à sa naissance, dans son état de détresse, hilflosigkeit.

Ce courage de penser me paraît inséparable d'une «politique » de la pensée, d'une politique qui refuse l'instrumentation radicale des conditions de 
penser, son impuissance symbolique et sa dévalorisation sociale. Façon comme une autre pour le politique de réaffirmer la spécificité de son propre champ, d'en revendiquer l'autonomie et de ne pas accepter de disparaître dans une "police des normes (Rancière, 2005) ", comme de se réduire au rôle de fondé de Pouvoir des intérêts du Marché globalisé. Comment ici ne pas penser à nouveau à Simone Weil écrivant: «On dit souvent que la force est impuissante à dompter la pensée ; mais pour que ce soit vrai, il faut qu'il y ait pensée. Là où les opinions irraisonnées tiennent lieu d'idées, la force peut tout. Il est bien injuste de dire par exemple que le fascisme anéantit la pensée libre; en réalité c'est l'absence de pensée libre qui rend possible d'imposer par la force des doctrines officielles totalement dépourvues de signification.» (1955, 142) Puissions-nous ne pas oublier l'aphorisme de Benjamin: «le conteur est la figure sous laquelle le juste se rencontre lui-même » (Benjamin, 1972/2000, 151).

\author{
Roland Gori \\ 101 rue Sylvabelle \\ F-13006 Marseille \\ roland.gori@orange.fr
}

\title{
Notes
}

1. Souligné par l'auteur.

2. Isonomie signifie " égalité» devant la loi.

3. Rapport sur la prise en charge du handicap psychique, Aout 2011 http://lesrapports. ladocumentationfrancaise.fr/BRP/114000570/0000.pdf

4. DSM : Diagnostic and Statistical Manual of Mental Disorders.

5. http://www.appeldesappels.org/

6. Tel est le sens du collectif de l'Appel des appels http://www.appeldesappels.org/

\section{Références}

ABELHAUSER, A. , GORI, R., et SAURET, M.-J., 2011, La folie évaluation Les nouvelles fabriques de la servitude, Paris, Mille et une Nuits-Fayard.

ADORNO, Theodor W., 1951, Minima Moralia Réflexions sur la vie mutilée, Paris, Payot, 2003. AGAMBEN, G., 2006, Qu'est-ce qu'un dispositif?, Paris, Payot \& Rivages, 2007.

AUSTIN, J.-L., 1970, Quand dire c'est faire, Paris, Seuil.

BECKER, G., 1964, Human Capital, Chicago, University of Chicago Press, 1994.

BENJAMIN, W., 1972, Euvres III, Paris, Gallimard, 2000.

BLECH, J., 2003, Les inventeurs de maladies, Arles, Actes Sud, 2005.

CANGUILHEM, G., 2011, Ecrits philosophiques et politiques 1926-1939. Euvres complètes tome 1, Paris, Vrin.

CANGUILHEM, G.,1947, « Note sur la situation faite en France à la philosophie biologique », Revue de métaphysique et de morale, 52 (3). 
DEL VOLGO, M.-J., 1997, L’instant de dire, Toulouse, Érès, 2012.

FOUCAULT, M., 1954, Maladie mentale et psychologie, Paris, PUF, 2005.

FOUCAULT, M., Les anormaux. Cours au Collège de France 1974-1975, Paris, Gallimard, 1999.

FREUD, S., 1939, L’homme Moïse et la religion monothéiste, Paris, Gallimard, 1986.

FREUD, S., BREUER, J., 1895, Études sur l'hystérie, Paris, PUF, 1956.

GORI, R., 2010, De quoi la psychanalyse est-elle le nom? Démocratie et subjectivité, Paris, Denoël.

GORI, R., 2011, La dignité de penser, Paris, LLL.

GORI, R., CASSIN, B., LAVAL, Ch. (dir.), 2009, Appel des appels. Pour une insurrection des consciences, Paris, Mille et une nuits.

GORI, R., DEL VOLGO, M.-J., 2005, La santé totalitaire, Paris, Flammarion, 2009.

GORI, R., DEL VOLGO, M.-J., 2008, Éxilés de l'intime, Paris, Denoël.

GORI, R., LE COZ, P., 2006, L'empire des coachs, Paris, Albin Michel.

HUGNET, G., 2004, Antidépresseurs. La grande intoxication, Paris, le Cherche-Midi.

LACAN, J., 1969-1970, Le séminaire livre XVII. L'envers de la psychanalyse, Paris, Seuil, 1991.

LYOTARD, J.-F., 1979, La condition postmoderne, Paris, Les éditions de minuit.

PIGNARRE, Ph., 2001, Comment la dépression est devenue épidémie, Paris, Hachette.

PIGNARRE, Ph., 2003, Le grand secret de l'industrie pharmaceutique, Paris, La Découverte.

RANCIERE, J., 2005, La haine de la démocratie, Paris, La Fabrique.

REY, A., (dir.), 1992, Dictionnaire historique de la langue française tome I, Paris, Dictionnaire le Robert.

REY, A., (dir.), 2005, Dictionnaire culturel de la langue française, Paris, Le Robert.

SIGERIST, H.-E., 1932, Introduction à la médecine, Paris, Payot.

VERNANT, J.-P., 1962, Les origines de la pensée grecque, Paris, PUF, 2007.

WEIL, S., 1955, Réflexions sur les causes de la liberté et de l'oppression sociale, Paris, Gallimard. 